\title{
KULTURELLE ASPEKTE VON TEXTSORTEN
}

1. Die Lehnbezeichnung "interkulturell" gehört zweifellos zu den jüngeren Lexemen, wenngleich sie der neue Duden, Jahrgang 1996, noch nicht verzeichnet. Auch von einer "gewissen Inflationierung" ihres Gebrauchs - so jedenfalls Lutz Götze (1992, 3) - kann man wohl nicht reden. Die Etablierung neuer Lehrbereiche und Lehrdisziplinen an deutschen Hochschulen mit den Bezeichnungen "Interkulturelle Germanistik",1 Konzepte interkulturellen Lernens, ${ }^{2}$ "Interkulturelle Hermeneutik"3 zeigt allenfalls Unsicherheiten in der semantischen Bestimmung des jungen Begriffs an. Es bleibt aber ein positiv konnotiertes Verständnis, das getragen ist vom Willen zum Dialog der Kulturen und vom Geist der Toleranz. Weit häufiger werden parallele Entwicklungen in der Weltwirtschaft verbalisiert. Die Rede ist von "Globalisierung", "globalisierter Wirtschaft" bzw. (in einem Gespräch der Zeitschrift "Deutschland" mit einem Bonner Politiker, Heft 5/96, S. 8) von "globalisierter Weltwirtschaft".

Kulturen umfassen bekanntlich alles im Leben menschlicher Gemeinschaften historisch Gewachsene wie auch das Aktuelle, gegenwärtig Gepflegte, d.h. Kunst und Wissenschaft, Gewohnheiten und Gebräuche, die Sprache in ihrem Werden und Sein, die materielle wie die geistige Produktion, Gesetze und Regeln im menschlichen Zusammenleben, Wertesysteme und die Art, sich zu verhalten. In dieser umfassenden Bestimmung tangieren sie auch ökonomische Entwicklungen. Kulturen sind anpassungs- und entwicklungsfähig, aber auch ebenso sensibel gegenüber Mißachtung und Intoleranz. Mit diesem Kulturverständnis ist die interkulturelle Kommunikation nicht zu begreifen als Kommunikation zwischen Menschen verschiedener kultureller

1 vgl. "Gesellschaft für interkulturelle Germanistik" wie auch das Studienfach "Interkulturelle Germanistik" an der Universität Bayreuth (nach Linguistik-Handbuch, hrsg. von Wilfried Kürchner, Tübingen 1994, S. 629 f.) - Das ist eine m. E. wenig geglückte (weil akollokative) Bildung, die partiell synonymisch für "Deutsch als Fremdsprache" steht.

2 Derlei Konzepte orientieren auf die Spezifik einer zu erlernenden Zweit- oder Drittsprache im Vergleich zur Erstsprache.

3 Zweifellos ist eine der kommunikativen Bedingungen zur Erklärung und zum Verständnis von Texten der kulturelle Hintergrund ihrer Entstehung. Zwar läßt sich der Begriff der Hermeneutik nicht nur im klassischen Sinne als "Methode des Verstehens" bzw. als "Verstehenslehre" definieren, sondern (z.B. bei K.O. Apel, nach H. Seiffert 12 f.) auch auf elementare schwer verständliche Aussagen anwenden, dennoch fällt es mir schwer, vor dem Hintergrund der HermeneutikGeschichte den Begriff der "interkulturellen Hermeneutik" zu akzeptieren. 
Bindungen in einer vereinbarten Sprache, sondern sie ist ethnisch wie auch ethisch angepaßtes Verhalten in der kommunikativen Interaktion.

Ich möchte daraus herleiten, daß "interkulturell" bzw. das entsprechende Substantiv zunächst ein Verhalten verbalisiert, z.B. durch ritualisierte Höflichkeitsbezeugungen in verschiedenen Kulturräumen. Dabei wird die Kenntnis der Rituale vorausgesetzt. Um die Kenntnis des historisch Überlieferten und Gewachsenen in einer fremden Kultur geht es auch, wenn Texte übersetzt und an fremde Textmuster angepaßt werden müssen. Ich komme darauf noch zurück.

Ganz anders zeigt sich das Interkulturelle in der sog. "Alltagshermeneutik", wie sie Helmut Seiffert einmal nannte $(1992,224)$. Hier geht es um das Verstehen bzw. Mißverstehen vor allem von Wendungen, deren Bedeutung oftmals bereits idiomatisiert ist. So wird beispielsweise die im Slowenischen häufig gebrauchte Höflichkeitsbezeugung izvoliti (in ihrer eigentlichen Bedeutung wählen, auserwählt sein) in der Bedeutung verwendet Bitte!, Bitte, nach Ihnen! wenn jemandem der Vortritt gelassen wird oder ihm eine Speise gereicht wird. Verläßt man am Abend ein Restaurant, grüßt man allenfalls beim Hinausgehen mit lahko noč, was übesetzt Gute Nacht! heißt. Im Deutschen würde man sich mit einem Auf Wiedersehen verabschieden, auch dann, wenn man gar nicht die Absicht oder vielleicht auch nicht den Wunsch hat, den Kellner oder einen zufälligen Tischnachbarn wiederzusehen. Es handelt sich um idiomatisierte Wendungen im zwischenmenschlichen Kontakt. Ebenso problematisch kann die Übersetzung von Termini in der interkulturellen fachsprachlichen Kommunikation sein. Zwar zeigt sich in den Fachsprachen eine Tendenz zur Internationalisierung. Aber es bleibt noch ein reicher Wortschatz, der mehr auf Vereinbarung und Überlieferung beruht als auf aktuellem Semantikverständnis. Schon der juridische Wortschatz in den deutschsprachigen Ländern Deutschland und Österreich weicht in vielem voneinander $a b$, weil er verschiedene historische Wurzeln hat. Ein österreichischer Minister wird beispielsweise angelobt, ein deutscher Minister vereidigt. Mit diesen Beispielen aus der Kommunikationspraxis referiert das Lexem "interkulturell" auf Unterschiede in der Benennungsmotivation. Es sind kulturspezifische semantische Gegebenheiten in der Alltags- und Fachkommunikation. Mit einer weiteren Bedeutung ist "interkulturell" auch zu beziehen auf den Austausch von Erfahrungen und Ideen, auf den Dialog von Kulturen, so daß folglich das noch recht junge Lexem bereits zu einem polysemen Wort mit positiver Konnotation geworden ist.

2. Im folgenden wird versucht, kulturelle Aspekte auch auf Textsorten zu beziehen. Kulturell deshalb, weil Textsorten bzw. Textmuster in ihrer kulturellen Tradition existieren. In einer fremdsprachlichen kommunikativen Situation werden sie der kulturellen Tradition angemessen, der auch die vereinbarte Sprache zuzuordnen ist. Die kulturelle Tradition von Textsorten kann für den Einzelfall in Frage gestellt werden. Das ist dann der Fall, wenn zumeist aus ökonomischen und verwaltungstechnischen Zwängen vornehmlich im Kommunikationsbereich der Direktive/ Administration Textmuster in der Form von Antragsformularen, Steuererklärungen, Vollmachten u.a.m. entstehen. Es sind vorgeschriebene Muster, die momentan als 
zweckmäßig angesehen werden und die nur insofern an Traditionen gebunden sind, als das Formularwesen in jedem Staatsgebilde seine Geschichte hat. Kulturprodukte sind sie allemal. Aber auch dieser sog. präskriptive Typus von Textsorten (vgl. S. Heusinger $1995,15)$ hat seine Vorbilder. Es ist eine neue Situation, eine neue situative Anforderung, die bisherige Interna des Textmusters verändert hat. G. Diewald verneint deshalb, daß Textsorten auch textexterne Merkmale hätten: "Vielmehr sind ihre 'textinternen' Merkmale, also die gesamten Textsortenmerkmale, von den 'textexternen', also situativen, Merkmalen bestimmt" (1991, 266).

Die Herausarbeitung kultureller Aspekte von Textsorten ist nicht unproblematisch, weil man nach dem Stand der Diskussion ${ }^{4}$ für die Musterbeschreibung - soweit sich daraus mehrfach Akzeptiertes herleiten läßt - mindestens auf folgende Merkmale schließen kann:

1. Die situative Einbettung (soweit sich aus der jeweils konkreten Situation für das einzelne Textexemplar ein situativer Rahmen für die Textsorte abstrahieren läßt. Ein Lebenslauf z.B. richtet sich meistens an eine Institution oder einen Betrieb. Folglich ist er hinsichtlich der sozialen Situation offiziell gebunden. Der Tätigkeitsbereich [Handlungsbereich] ist zumeist die Direktive/Administration, woran die Erwartung geknüpft ist, daß er authentisch und sachlich ist; die Situation ist auch durch die Kommunikationsrichtung und den Kanal charakterisiert: dialogisch, monologisch; mündlich, schriftlich, fernmündlich)

2. Die dominierende Intention bzw, die kommunikative Zwecksetzung. Sie korreliert mit der Textsortenfunktion (ein Lebenslauf will informieren, ein Kochrezept anleiten, unterweisen [instruieren])

3. Das generelle Thema (als der Leitgegenstand, als die gesellschaftlich erwartete Grundidee)

4. Sprache/Stil (erwartbare sprachliche Existenzform und Stilschicht: z.B. Standardsprache, gehoben oder normal; Musterhaftes in der Stilgestaltung)

5. Komposition (Themaentfaltung, Anordnung der Inhalte, z.B. für einen Lebenslauf chronologische oder thematische Anordnung)

$\mathrm{Zu}$ fragen ist, ob alle diese Merkmale auf jede Art von Textsorten bezogen werden können. Ist beispielsweise das Sprichwort eine "Textsorte"? Nach M. Wierschin sind Textsorten nicht als komplexe Muster sprachlicher Handlungen $\mathrm{zu}$ definieren, sondern als "feste clichéhafte Denk- und Aussageschemata, als vorgefertigte Formeln, Phrasen, Zitate, Wendungen" (...) auch als "Phraseologismen und Sprichwörter in Einzeltexten" $(1995,69)$. Es sind - wie M. Wierschin gleichfalls betont - "stilistische Fertigteile" $(1995,65)$. Nun sind Textsorten in der Tat keine Texte, sondern textuelle Muster, die als "wichtige Entscheidungsinstanz für Effizienz, Effektivität und Angemessenheit" dienen können (R.-A. de Beaugrande/ W.U. Dressler 1981, 193). Aber sie implizieren das semantische Merkmal "textuell", was für Phrasen, Formeln, Wendungen, also für

4 Einen weitgreifenden. Überblick über den Forschungsstand zur Beschreibung von Textsorten gibt G. Diewald in ihrem Buch "Deixis und Textsorten im Deutschen", 1991, $272 \mathrm{ff}$. 
elementare Muster auf der Satzebene nicht zutrifft, obwohl es - wie bekannt - auch Texte gibt, die aus weniger als einem Satz bestehen. Die aus diesen Überlegungen entstandene Problematik kann auch nicht mit einem Hinweis auf etwa unzulängliche zeichentheoretische oder handlungstheoretische Textdefinitionen erklärt werden. Es sind offensichtlich randlinguistische Phänomene. Sprichwörter, selbst Wortgruppen, können erklärtermaßen als Texte angesehen werden, wenn sie als Produkte sprachlich-kommunikativer Handlungen in sich geschlossene thematische Einheiten bilden. Nur lassen sich beispielsweise Sprichwörter nicht auf ein formales Text- und Strukturmuster zurückführen. Aber sie folgen einem Intentionsmuster, denn bei weitgehender Generalisierung ihrer Sinnhaftigkeit vermitteln sie bewahrte Volksweisheiten, die mit persuasiver oder belehrender Funktion in der Kommunikation gewählt werden. Daraus ist zu schließen, daß (1) der Textsortenbegriff sehr weit oder sehr eng definiert werden kann (je mehr Textsortenmerkmale/Invarianten angenommen werden, desto enger wird der Textsortenbegriff) und (2) daß bei bekannter Dualität von funktionaler Varianz und freier Varianz im Textgestaltungsprozeß die freie Wahl der Mittel in den Überlegungen zur Anwendung der Muster in sprachlichen Handlungen noch mehr Aufmerksamkeit bei ihrer wissenschaftlichen Behandlung erfordert.

Ungeachtet ihres Ursprungs oder ihrer Geschichte erweisen sich Textsorten als kulturelle Entitäten. Für die Kommunikationsteilnehmer sind sie in der zwischenmenschlichen Verständigung erfahrene, weil überlieferte, zumeist unterbewußt gespeicherte Orientierungsmuster, die mehr oder weniger Entscheidungen der Kommunizierenden im Sprachgestaltungsprozeß steuern. Mit dem Verständnis, Textsorten als Erfahrungen zu begreifen, die im Alltagsbewußtsein bewahrt sind, sind sie Elemente einer Kultur, die dem Einzelnen wie der ganzen Nation gehören.

Allerdings ist das National- bzw. Kulturspezifische in vergleichbaren Textsorten verschiedener kultureller Herkunft, das uns in der fremdsprachlichen Kommunikation sehr interessiert, vornehmlich in traditionellen Formulierungs- und Gestaltungsmustern zu suchen. Andere Textsortencharakteristika wie die situative Einbindung, die kommunikative Zwecksetzung, die thematische Bindung und die Komposition haben wie ich behaupten kann - kaum eine kulturspezifische Prägung. Für gesicherte Aussagen fehlen jedoch noch Untersuchungen. Unterschiede in der Formulierung und Textgestaltung, die mit verschiedenen Sprachsystemen erklärt werden müssen, gründen sich auf verbalisiertes Verhalten, z.B. durch Gruß- und Anredeformen, durch die Verbalisierung von Distanz, Offizialität, Zuneigung, Rollenzuweisung. Im Traditionellen verhaftet ist auch der Grad der Informationsdichte (z.B. zwischen slowenischen und deutschen sog. ausführlichen Lebensläufen). Selbst die Geschlechterrolle mit der heute überlebten Auffassung, die Frau allein sei für die Küche zuständig, kann ein verbalisiertes Element des Textmusters sein, wie das beispielsweise noch im vergangenen Jahrhundert in slowenischen Kochrezepten der Fall war.

Das Formulierungs- und Gestaltungsprofil des Textes - nennen wir es Textsortenstil - ist (wie W. Fleischer, G. Michel und G. Starke 1993, 35 betonen) nicht über einzelne Stilelemente des für eine Textsorte charakteristischen Stils beschreibbar, 
sondern über sog. Stilwerte. So kann beispielsweise durch die bevorzugte Verwendung von Indefinitpronomina, Passivkonstruktionen und dem erweiterten Inifintiv ein hoher Grad an Unpersönlichkeit für eine spezifische Textsorte realisiert und sozial erwartet werden. Das Unpersönliche im Ausdruck ist der Stilwert, der für einen bestimmten Textsortenstil kennzeichnend ist. Auch der o.g. Grad unterschiedlicher Informationsdichte für slowenische und deutsche Lebensläufe ist als Stilwert anzusehen.

Beim Vergleich deutscher und slowenischer Textsorten ist von einer Affinität auszugehen. Einige wesentliche Gründe dafür liegen wohl

- $\quad$ in der zeitweilig gemeinsamen Geschichte mit der Habsburger Monarchie und der daraus resultierenden gegenseitigen Befruchtung der Kulturen, was die jeweils eigenen Wurzeln, eigenen Lebenshaltungen, eigenen Gefühle aber nicht in Frage stellt bzw. jemals in Frage gestellt hat,

- im Trend zur Internationalisierung der Textsorten, der mit den immer enger werdenden Bindungen der Völker in allen Lebensbereichen korreliert,

- im entfalteten Dialog der Völker mit Unterstützung der Medien, der Reisetätigkeit und zwischenstaatlich geförderter Maßnahmen,

- $\quad$ in der Eingliederung in die zum Beispiel europäische Kultur, der gemeinsame Stile, gemeinsame historische Epochen, gemeinsame aktuelle Fragen inhärent sind.

In diesem Spezialfall möchte ich von "affinen Textsorten" sprechen. Sie verhalten sich zueinander wie verwandte Personen, die dennoch verschiedene Individuen sind. Verwandtes im geschichtlichen Prozeß und Trennendes liegen dicht beieinander. Das "kulturell Einmalige" in deutschen und slowenischen Brief-Textsorten (und nicht nur für diese Sprachen), wenn man es nicht diachronisch betrachtet, zeigt sich auffällig in den Anrede- und Grußformen.

Nur geringe Unterschiede bestehen in der offiziellen schriftlichen Kommunikation, wie aus nachstehender Übersicht hervorgeht:

\begin{tabular}{|l|l|l|}
\hline $\begin{array}{l}\text { Sehr geehrte Damen und Herren } \\
\text { Sehr geehrte Damen, sehr gee- } \\
\text { hrte Herren }\end{array}$ & $\begin{array}{l}\text { Spoštovani, } \\
\text { Spoštovane dame, } \\
\text { Sehr geehrter Herr Meyer } \\
\text { Sehr geehrter Herr Professor } \\
\text { Spoštovani gospodje, } \\
\text { Spoštovani/cenjeni gospod } \\
\text { profesor }\end{array}$ & $\begin{array}{l}\text { ohne Damen, Herren } \\
\text { nur in mündlicher } \\
\text { "cenjeni" klingt veraltend }\end{array}$ \\
Nur beigleichem Status & Dragi gospod Majer & nur selten verwendet \\
\hline Lieber Herr Meyer & $\begin{array}{l}\text { Prisrčen pozdrav/ Prisrčne } \\
\text { pozdrave } \\
\text { Lepe pozdrave/ Lep pozdrav } \\
\text { Sit freundlichem Gruß/ freuntovanjem }\end{array}$ & bei gleichem Sozialstatus \\
dlichen Grüßen & bei ungleichem Sozialstatus \\
Hochachtungsvoll & & \\
\hline
\end{tabular}


Speziellere Anrede- und Grußformen z. B. im Schriftverkehr mit Ministern, Botschaftern habe ich nicht berücksichtigt. Der Gruß Prisrčen (herzlich) ist im deutschen offiziellen Schriftverkehr nicht zu finden. In slowenischer offizieller schriftlicher Kommunikation entsprechen die "herzlichen Grüße" den deutschen Grußformen Mit freundlichen Grïßen/Mit besten Grïßen. Es werden besonders gute, dennoch offizielle Beziehungen zu sozial Gleichgestellten angezeigt. Auch in persönlichen Briefen ist die Form Herzliche Grïße im slowenischen Briefverkehr zwischen nahen Verwandten mit sehr guter inniger Beziehung zu finden, z.B. in der Form Prisrčno te pozdravlja tvoja... (Sei herzlich gegrüßt von Deiner ...). Der Gebrauch des Adjektivs herzlich in slowenischer offizieller wie auch intim-verwandtschaftlicher Kommunikation zeigt weniger die Besinnung auf die konnotative Semantik an als vielmehr den rituellen Gebrauch. Es sind Formeln in Umgangs- und GruBritualen, die zu unserer Kultur gehören und die wir pflegen. Viele dieser Formeln beispielsweise im offiziellen Briefkontakt zu Institutionen sind zu leeren Formeln verblaßt. Dazu gehört zweifellos im Deutschen die Anrede Sehr geehrte Damen und Herren und der Briefabschluß Mit freundlichem Gruß. Dennoch haben diese Formeln eine Funktion. Sie sind notwendige rahmenbildende Elemente eines Textmusters. Fehlen sie oder weichen sie von der erwarteten Form ab, werden positive oder (zumeist aber) negative Effekte hervorgerufen. Man stelle sich eine schriftliche Mahnung des Finanzamtes mit der Anrede Lieber Steuerzahler vor. Es wäre ungewöhnlich schön.Vom Unerwarteten ausgehende Effekte sind in interkultureller Kommunikation in der Regel nicht beabsichtigt, aber sie werden gern verziehen.

Wie beständig Überliefertes in ritualisierten Formeln ist, zeigt ein Antrag zur Vermeidung sexistischen Sprachgebrauchs 1992 an das niedersächsische Landesgericht Hannover. Beantragt wurde, die offizielle Anrede Sehr geehrte Frau Meyer durch die amtliche Anrede Sehr geehrte Dame Meyer zu ersetzen. Begründet wurde der Antrag mit der gebräuchlichen analogen männlichen Form Sehr geehrter Herr Meyer. Es war davon ausgegangen worden, daß das weibliche Pendant zu Herr nicht "Frau" sondern Dame sei. Die Antragstellerinnen waren zweifellos im Recht, doch der Antrag mußte mit dem Hinweis auf geläufige Anredeformeln zurückgewiesen werden.

Auffällig beim Vergleich von Anredeformen im Slowenischen und Deutschen ist die situativ bedingte Vermeidung des im Deutschen wohl häufigsten Anredeadjektivs liebe in solchen Verbindungen Lieber Herr Dr. Meyer; Liebe Mutter (Mutti), Liebes Fräulein Sonja, lieber Papa u.a.m. Die Anrede Ljubi gospod dr. Mayer ist völlig undenkbar, weil das Adjektiv ljuba im Slowenischen auf eine sehr intime Beziehung verweist und deshalb anders als das deutsche Adjektiv konnotiert ist. Entsprechend ist es auch in seiner Extension mit Bezug auf Personen wesentlich enger als das deutsche Adjektiv liebe. Mit Bezug auf Gegenstände, Sachen ist es in seiner Extension wiederum weiter anwendbar, z.B. in der Bedeutung "wertvoll", "teuer" wie das ljubi kruhek (das liebe Brot). Im Slowenischen wäre es sehr ungewöhnlich, den leiblichen Vater in einem Brief mit Ljubi ati oder Ljubi očka anzureden. Hingegen ist es üblich, 
dafür das Adjektiv drag zu verwenden, welches die Bedeutung "wertvoll", "teuer" mit Bezug auf Personen trägt, also dragi ati bzw. dragi očka.

Ich fasse meine Überlegungen zu kulturellen Aspekten von Textsorten zusammen:

1. Mit unserem Kulturverständnis ist die terminologisierte Wortgruppe "interkulturelle Kommunikation nicht zu begreifen als Kommunikation zwischen Menschen verschiedener kultureller Bindungen, sondern sie ist ethnisch wie auch ethisch angepaßtes sprachlich-kommunikatives Verhalten an kulturelle Traditionen, der auch die vereinbarte Sprache unterliegt.

2. Das Adjektiv "interkulturell" referiert auf idiomatisierte Wendungen im zwischenmenschlichen Kontakt wie auch auf kulturell gebundene Unterschiede in der Benennungsmotivation

3. Das Adjektiv "interkulturell" ist auch zu beziehen auf den Austausch von Erfahrungen und Ideen, auf den Dialog von Kulturen.

4. Textsorten existieren in ihrer kulturellen Tradition, wobei das Kulturspezifische vornehmlich in traditionellen (aber entwicklungsoffenen) Formulierungs- und Gestaltungsmustern zu suchen ist. Andere Textsortencharakteristika wie die situative Einbettung, die kommunikative Zwecksetzung, die thematische Bindung und die Komposition haben offenbar kaum eine kulturspezifische Prägung.

5. Im Trend zur Internationalisierung der Textsorten erweisen sich die kulturell geprägten Textmuster vor allem zwischen angrenzenden Nationen häufig als affin.

\section{Literatur:}

de Beaugrande, Robert/Dressler, Wolfgang Ulrich: Einführung in die Textlinguistik. Tübingen 1981

Diewald, Gabriele: Deixis und Textsorten im Deutschen. Tübingen 1991

Götze, Lutz: Interkulturelles Lernen und "Interkulturelle Germanistik" - Konzepte und Probleme. In: Deutsch als Fremdsprache, 29. Jahrgang, Heft 1, München/Berlin 1992, Seiten 3 bis 9

Heusinger, Siegfried: Textsorten in der interkulturellen Kommunikation. - Ein Problemaufriß. In: Besedilne vrste - Textsorten. Hrsg. von S. Heusinger und A. Janko, Zeitschrift "Linguistica". Heft XXXV,1. Ljubljana 1995, Seiten 7 - 20

Seiffert, Helmut: Einführung in die Hermeneutik. Tübingen 1992

Wierschin, Martin: Sprache, Text und Textsorten: Zur Problematik von Texttypologie. In: Besedilne vrste - Textsorten. Hrsg. v. S. Heusinger und A. Janko, Zeitschrift "Linguistica". Heft XXXV,1. Ljubljana 1995, Seiten 53 - 79 


\section{Zusammenfassung}

Ich fasse meine Überlegungen zu kulturellen Aspekten von Textsorten zusammen:

1. Mit unserem Kulturverständnis ist die terminologisierte Wortgruppe "interkulturelle Kommunikation" nicht zu begreifen als Kommunikation zwischen Menschen verschiedener kultureller Bindungen, sondern sie ist ethnisch wie auch ethisch angepaßtes sprachlichkommunikatives Verhalten an kulturelle Traditionen der jeweils für den Kommunikations- akt vereinbarten Sprache.

2. Das Adjektiv "interkulturell" referiert auf idiomatisierte Wendungen im zwischenmenschlichen Kontakt wie auch auf kulturell gebundene Unterschiede in der Benennungsmotivation (z.B. anloben /österr./, vereidigen $/ \mathrm{dt} . /)$

3. Das Adjektiv "interkulturell" ist ebenfalls zu beziehen auf den Austausch von Erfahrungen und Ideen, auf den Dialog von Kulturen.

4. Textsorten existieren in ihrer kulturellen Tradition, wobei das Kulturspezifische vornehmlich in traditionellen (aber entwicklungsoffenen) Formulierungs- und Gestaltungsmustern zu suchen ist. Andere Textsortencharakteristika wie die situative Einbettung, die kommunikative Zwecksetzung, die thematische Bindung und die Komposition haben offenbar kaum eine kulturspezifische Prägung.

5. Im Trend zur Internationalisierung der Textsorten erweisen sich die kulturell geprägten Textmuster vor allem zwischen angrenzenden Nationen häufig als affin.

\section{Povzetek \\ KULTURNI VIDIKI BESEDILNIH VRST}

Svoja razmišljanja o kulturnih vidikih besedilnih vrst povzemam takole:

1. Terminologizirane besedne zveze "medkulturna kornunikacija" ne moremo z našim razumevanjem kulture razumeti kot sporazumevanje med ljudmi, vezanih na različne kulture, temveč gre za jezikovnosporazumevalno vedenje, ki je etnično kot tudi etično prilagojeno kulturnim tradicijam vsakokratnega, za sporočanjsko dejanje izbranega jezika.

2. Pridevnik "medkulturni" se nanaša na na frazeme v medčloveškem stiku kot tudi na kulturno vezane razlike v motivacijah za poimenovanje (npr. anloben /avstr./, vereidigen /nem./) kulturami.

3. Pridevnik "medkulturni" je prav tako treba navezati na izmenjavo izkušenj in idej, na dialog med

4. Besedilne vrte obstajajo $v$ kulturni tradiciji, pri cemer je treba specificno kulturno iskati predvsem v tradicionalnih (a razvoju odprtih) vzorcih formulacij in oblik. Druge besedilnovrstne karakteristike kot tudi situacijska vpetost, komunikacijski namen, tematska vezanost in kompozicija imajo očitno komajda kulturnospecifični značaj.

5. Glede na trend internacionalizacije besedilnih vrst se kulturno zaznamovani besedilni vzorci pogosto pokažejo kot sorodni predvsem med sosednjimi narodi. 